\title{
Psychiatric manifestations as the leading symptom in an expatriate with dengue fever
}

\author{
J. A. Blum • S. Pfeifer $\cdot$ C. F. Hatz
}

Received: 29 January 2010/Accepted: 3 May 2010/Published online: 22 June 2010

(c) Urban \& Vogel 2010

A 52-year-old Swiss female patient suffered from intermittent fever up to $39^{\circ} \mathrm{C}$, headache, moderate diarrhea and dizziness within 2 months after arriving in Haiti where she worked for a non-governmental organization. She collapsed three times but regained consciousness immediately after being placed in a lying position. Although the patient interpreted these events as resulting from a mild flu-like illness combined with orthostatic collapse, she was hospitalized 3 days later to rule out underlying cardiac disease. The initial clinical examination showed hypotension (blood pressure $80 / 60 \mathrm{mmHg}$ ), bradycardia (pulse rate 40 beats per minute) and severe fatigue (Fatigue Severity Score 6-7). The day following her admission to hospital, she developed a psychiatric syndrome characterized by the following symptoms: ideas of reference and delusions with vivid acoustic and visual hallucinations, accompanied by agitation and psychotic fears. She had the impression that part of the staff was trying to harm her, as she heard voices denouncing her from people close to a (non-existent) swimming pool. She also had the impression that there was

\footnotetext{
J. A. Blum $(\bowtie) \cdot$ C. F. Hatz

Swiss Tropical and Public Health Institute, Socinstrasse 57, 4002 Basel, Switzerland

e-mail: johannes.blum@unibas.ch

C. F. Hatz

e-mail: christoph.hatz@unibas.ch

S. Pfeifer

Psychiatric Clinic Sonnenhalde, Chrischonaweg 50,

4125 Riehen, Switzerland

e-mail: samuelpfeifer@gmx.net

C. F. Hatz

Institute of Social and Preventive Medicines,

University of Zürich, Zurich, Switzerland
}

writing on the bed linen, red in color, and that this writing was a list of judgmental comments on her (mis)behavior. To counteract this "judgments", she tore the linen and began to write her "defense" on the linen using a pen with black ink. The intravenous drip intended to rehydrate the patient was another object of psychotic fears: she hallucinated embryos in the saline solution and therefore tried to tear out the drip. During the night she suffered from nightmares in which she mixed childhood experiences with the grim reality of poverty in Haiti. All of these hallucinations resulted in a fluctuating emotional state that ranged from fear and sadness to anger and a call to action. When addressed by her husband and staff, she was able to answer simple questions, and she did not refuse eating and drinking. Fever was not a leading symptom during the hospitalization period, and it subsided after a few days.

The laboratory tests revealed a leucopenia of $4,000 / \mathrm{mm}^{3}$ at admission and $2,000 / \mathrm{mm}^{3}$ the following day (normal value $4,000-10,000 / \mathrm{mm}^{3}$ ), slightly elevated values for both the liver tests and creatine phosphokinase. Slides for malaria tested negative, and the results of the analysis of the urine were normal. A 24-h electrocardiography and echocardiography showed normal results.

The treatment included rehydration with intravenous and oral fluids as well as vitamin and iron supplementation. The psychosis was treated with oral doses of lorazepam and the antipsychotic drug risperidone and a single injection of long-acting fluphenazine, all of which had a marked sedative effect. The hallucinations and delusions vanished over the next 3 days, and the general condition of the patient improved satisfactorily over 6 days. No signs of bleeding were observed at any stage of the disease, but the patient had a strong menstruation. A viral disease or encephalitis were considered as a possible diagnosis. The patient was discharged on risperidone medication. 
The psychiatric syndromes were a cause of concern to our patient's employer. She was therefore repatriated to Switzerland and referred to our outpatient clinic (Tropical Medicine) and to a psychiatric consultant for an assessment of her ability to continue her working stay in Haiti. The patient had no previous psychiatric history and no family history of psychosis.

Six weeks after the onset of the symptoms the patient was examined in our clinics. She complained about weakness, gaze difficulties and slightly trembling hands. The physical examination, including the neurological and psychiatric assessment, revealed no findings of significance. Risperidone was discontinued, and there was no relapse of psychotic symptoms over the following months.

The laboratory values, including the white blood cell count, platelet count and hemoglobin level as well as those for the liver tests and kidney function tests, were all normal. Serologic testing (IIFT) showed highly elevated titers of immunoglobulin M (IgM; 1:640) and $\operatorname{IgG}(1: 10,240)$ antibodies against dengue virus. However, cross-reaction with other members of the genus Flavivirus, such as those of yellow fever, Japanese encephalitis, tick-borne encephalitis or St Louis encephalitis, may interfere with dengue IgG antibodies [1, 2]. The patient had been vaccinated against tick-borne encephalitis 3 years prior, but never against yellow fever and Japanese encephalitis. The serologic testing against tick-borne encephalitis showed positive antibodies IgG IIFT (1:640) and negative IgM antibodies, compatible with the vaccination 3 years before. The antibodies against St. Louis encephalitis (IIFT) were negative for $\operatorname{IgM}$ and slightly positive for $\operatorname{IgG}(1: 160)$, compatible with a cross-reaction to dengue virus. The diagnosis of dengue in this patient was therefore suspected based on clinical manifestations, travel history and leucopoenia/thrombopenia and subsequently confirmed by the presence of high levels of positive IgM and IgG antibodies after exclusion of the cross-reactions.

A psychotic reaction as leading symptom, which ultimately caused the return of an expatriate employee, is an unexpected and unique aspect of dengue fever. Other symptoms of encephalitis, such as convulsions, somnolence or neurological deficit, were not observed in this patient, and the febrile disease was not prominent. To the best of our knowledge, this is the first detailed description of a patient with dengue fever and psychotic symptoms.

Neurological and psychiatric involvement in dengue patients can be due to the infection itself with viral encephalitis or be secondary to metabolic disturbances. In our patient, the laboratory results did not reveal any evident disturbance, but a metabolic disorder could not be ruled out because tests for electrolytes and other parameters were not performed in Haiti.
The incidence of neurological and psychiatric symptoms in dengue fever in adults is difficult to estimate because neurological manifestations have only been described in selected compilations of specialized referral clinics $(21 \%)$ [3], case series [4-6] or case reports [7-10]. Among 1,493 Thai children admitted to hospital, the incidence of neurological symptoms was $5.4 \%$ [11]. It is probable that the incidence in an unselected population would be much lower since neurological symptoms have not yet been reported in large series [12-14]. The main neurological symptoms reported to date include alteration of consciousness, seizures, mental confusion and nuchal rigidity. Other neurological deficits include spasticity of the limbs, hemiplegia, quadriparesis and abnormal coordination [3-5, 11].

Psychiatric symptoms or mental confusion are usually described as part of an encephalitic syndrome together with alteration of consciousness, neurological deficit or seizures [3-5, 11]. Dengue fever is usually suspected based on its typical clinical picture that includes fever. However, similar to the history of our patient, in one study, the characteristic features of dengue were not present in 12 of 21 dengue patients with neurological symptoms at admission [4]. Psychiatric manifestations as the leading symptom have been described in case reports [8, 9], but hallucinations have not been mentioned. Confusion, abnormal behavior or mental disorder as a single neurological symptom was mentioned-but only rarely - in a case series, but a detailed description of the psychiatric symptoms was not given [4].

The case reported here illustrates that a psychiatric symptom may be the leading symptom in patients with dengue fever. As such, it may be misinterpreted in the first consultation and should be considered in any patient with fever and neurological or psychiatric symptoms within 14 days after a stay in an endemic area, even if the fever is not a key symptom.

Conflict of interest statement None.

\section{References}

1. Wilder-Smith A, Schwartz E. Dengue in travelers. N Engl J Med. 2005;353:924-32.

2. Allwinn R, Doerr HW, Emmerich P, Schmitz H, Preiser W. Cross-reactivity in Flavivirus serology: new implications of an old finding? Med Microbiol Immunol. 2002;190:199-202.

3. Domingues RB, Kuster GW, Onuki-Castro FL, Souza VA, Levi JE, Pannuti CS. Involvement of the central nervous system in patients with dengue virus infection. J Neurol Sci. 2008;267:36-40.

4. Solomon T, Dung NM, Vaughn DW, Kneen R, Thao LT, Raengsakulrach B, et al. Neurological manifestations of dengue infection. Lancet. 2000;355:1053-9.

5. Misra UK, Kalita J, Syam UK, Dhole TN. Neurological manifestations of dengue virus infection. J Neurol Sci. 2006;244:117-22. 
6. Matlani M, Chakravarti A, Rawal A, Kashyap B, Gurtoo A. Dengue encephalitis: an entity now common in dengue-prone regions. Trop Doct. 2009;39:115-6.

7. Angibaud G, Luaute J, Laille M, Gaultier C. Brain involvement in Dengue fever. J Clin Neurosci. 2001;8:63-5.

8. Mendhekar DN, Aggarwal P, Aggarwal A. Classical mania associated with dengue infection. Indian J Med Sci. 2006;60:115-6.

9. Rapp C, Debord T, Imbert P, Roue R. A psychiatric form of dengue after a visit to Djibouti. Presse Med. 2002;31:1704.

10. Nilsson J, Vene S, Mattsson L. Dengue encephalitis in a Swedish traveller returning from Thailand. Scand J Infect Dis. 2005;37: 776-8.

11. Pancharoen C, Thisyakorn U. Neurological manifestations in dengue patients. Southeast Asian J Trop Med Public Health. 2001;32:341-5.
12. Kalayanarooj S, Vaughn DW, Nimmannitya S, Green S, Suntayakorn S, Kunentrasai N, et al. Early clinical and laboratory indicators of acute dengue illness. J Infect Dis. 1997;176:313-21.

13. Hanafusa S, Chanyasanha C, Sujirarat D, Khuankhunsathid I, Yaguchi A, Suzuki T. Clinical features and differences between child and adult dengue infections in Rayong Province, southeast Thailand. Southeast Asian J Trop Med Public Health. 2008;39: 252-9.

14. Harris E, Videa E, Perez L, Sandoval E, Tellez Y, Perez ML, et al. Clinical, epidemiologic, and virologic features of dengue in the 1998 epidemic in Nicaragua. Am J Trop Med Hyg. 2000; 63:5-11. 